\title{
Human Migration
}

National Cancer Institute

\section{Source}

National Cancer Institute. Human Migration. NCI Thesaurus. Code C15422.

Movement of residence from one place to another. Includes nomadism; labor or seasonal migration; patterns of rural, urban, or suburban migration; or voluntary or forced relocation. (PSY94) 